\title{
Germination et établissement de semis naturels d'épinette rouge (Picea rubens) dans des trouées sylvicoles de différentes tailles
}

\author{
par Daniel Dumais ${ }^{1,2}$ et Marcel Prévost ${ }^{1}$
}

\begin{abstract}
RÉSUMÉ
Lépinette rouge (Picea rubens Sarg.) est difficile à régénérer par ensemencement naturel après une intervention sylvicole. Pour étudier sa germination et son établissement, nous avons suivi durant 10 ans la dynamique des nouveaux semis dans des trouées sylvicoles de différentes tailles (petites : $<100 \mathrm{~m}^{2}$, moyennes : 100-300 $\mathrm{m}^{2}$, grandes : $700 \mathrm{~m}^{2}$ ). La densité de semis a été plus élevée dans les petites trouées, mais le taux de survie n’a pas dépassé $40 \%$, laissant peu de semis vivants après 10 ans $\left(<200 \mathrm{ha}^{-1}\right)$. Les buttes ont été la meilleure microtopographie pour les semis. Nos résultats confirment le rôle important du bois en décomposition et de la mousse pour la germination et létablissement sous couvert. Le bois pourri s'est avéré important pour l'établissement dans les grandes trouées, alors que l'humus a été plus favorable dans les moyennes trouées. Dans les petites trouées, les germinants et les semis établis se trouvaient autant sur de la mousse que sur de l'humus ou du bois pourri. Cependant, les faibles densités observées suggèrent que la plantation dans de petites ou moyennes trouées soit une avenue à explorer afin d’accélérer le renouvellement de lespèce, d’autant plus si la régénération préétablie est déficiente.
\end{abstract}

Mots-clés : Bois en décomposition, épinette rouge (Picea rubens), lits de germination, microsites, régénération naturelle, trouées sylvicoles.

\section{ABSTRACT}

Red spruce (Picea rubens Sarg.) is difficult to regenerate from natural seeding following a silvicultural treatment. In order to study its germination and establishment, we monitored the dynamics of new seedlings during 10 years in silvicultural gaps of different sizes (small: $<100 \mathrm{~m}^{2}$, medium: 100-300 $\mathrm{m}^{2}$, large: $700 \mathrm{~m}^{2}$ ). Seedling density was higher in small gaps, but survival rate did not exceed $40 \%$, leaving few living seedlings after 10 years $\left(<200 \mathrm{ha}^{-1}\right)$. Mounds were the best microtopography for the seedlings. Our results confirm the important role of decaying wood and moss for understory germination and establishment. Decaying wood was important for the establishment in large gaps, while humus was more favourable in medium gaps. In small gaps, germinants and established seedlings were found as much on the moss as on the humus or decaying wood. However, low observed densities suggest that plantation in small or medium silvicultural gaps should be explored for accelerating species renewal, especially if advance regeneration is deficient.

Keywords: Decaying wood, microsites, natural regeneration, red spruce (Picea rubens), seedbeds, silvicultural gaps.

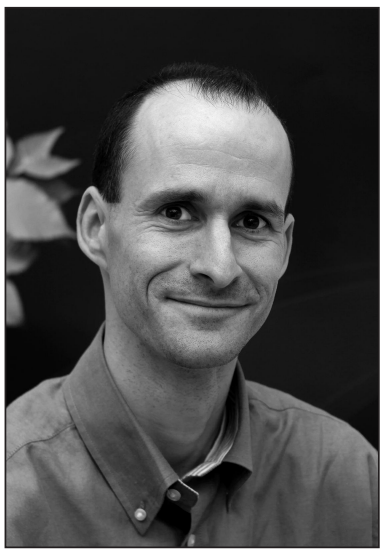

Daniel Dumais

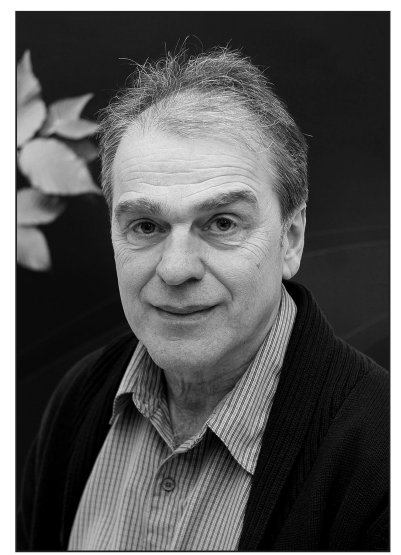

Marcel Prévost

\section{Introduction}

Pouvant atteindre $25 \mathrm{~m}$ de hauteur, $60 \mathrm{~cm}$ de diamètre et près de 400 ans, l'épinette rouge (Picea rubens Sarg.) est une essence forestière commune dans le nord-est de l'Amérique du Nord (Farrar 1996). Son bois, dense et résistant, est depuis longtemps très apprécié (Dumais et Prévost 2013). Ainsi, les peuplements contenant de lépinette rouge ont toujours subi beaucoup de pression de la part de l'industrie forestière. Par le passé, la sylviculture nétait pas forcément axée sur le maintien de cette essence compagne, d'autant plus que, de par son autécologie particulière, lépinette rouge est difficile à régénérer par ensemencement naturel après une intervention sylvicole (Dumais et Prévost 2007). En conséquence, la raréfaction connue de cette espèce constitue aujourd'hui un enjeu économique, mais aussi de biodiversité en ce qui a trait à la composition forestière (Grondin et Cimon 2003).

\footnotetext{
${ }^{1}$ Ministère des Forêts, de la Faune et des Parcs du Québec, Direction de la recherche forestière, Service de la sylviculture et du rendement des forêts, 2700, rue Einstein, Québec (Québec) G1P 3W8, Canada

${ }^{2}$ Auteur correspondant Courriel : daniel.dumais@mffp.gouv.qc.ca
} 
Les bonnes années semencières de l'épinette rouge ne se produisent qu'aux 3 à 8 ans (White et Cogbill 1992) et la banque de semences dans le sol est inexistante (Cavallin et Vasseur 2008). La viabilité des graines est faible (max. 12 mois; Morgenstern et Farrar 1964) et celles-ci sont prisées des petits mammifères malgré qu’elles soient très petites avec peu de réserves nutritives. Pour survivre, les plantules doivent donc pouvoir accéder aisément à de l'eau et des nutriments. Or, leur croissance racinaire est lente et les racines sont fines (Klein et al. 1991, Greenwood et al. 2008), ce qui se traduit par la fragilité des jeunes semis, une faible croissance initiale et un taux élevé de mortalité. Les semis sont par ailleurs vulnérables à l'écrasement par la litière feuillue (Place 1955). Ainsi, à ce stade, l'épinette rouge s'avère peu compétitive, d’où l'importance de la microtopographie et de la nature du lit de germination. Selon certaines observations, les microsites surélevés, comme les vieilles souches et les gros débris ligneux en décomposition, seraient favorables à la germination et possiblement à l'établissement des semis d'épinette rouge (Perkins 1991, Smallidge et Leopold 1994, Weaver et al. 2009). Ces microsites conservent l'humidité, accumulent peu de litière et permettent aux semis de sélever au-dessus de la végétation concurrente. Enfin, le broutement par les cervidés est également un facteur à considérer (Kenefic et al. 2014).

Lépinette rouge est très tolérante à l'ombre. Elle peut survivre en sous-bois pendant des décennies et reprendre une croissance vigoureuse à la faveur d'une ouverture partielle du couvert forestier (Seymour 1995). Cet aspect fait partie de sa stratégie de régénération, qui consiste à établir, au fil du temps, une banque de semis et de gaules en sous-étage. Pour survivre sous le couvert, l'épinette rouge est dotée d'une grande tolérance physiologique et d'une capacité d'ajustement morphologique à la faible lumière (Dumais et Prévost 2007). En contrepartie, cette adaptation ne lui permet pas de s'acclimater rapidement au changement brusque des conditions environnementales (Dumais et Prévost 2008). Ainsi, le degré et la vitesse douverture du couvert forestier sont importants dans le processus de régénération de l'espèce. Cependant, les conditions de microsites et d'ouverture du couvert favorables à la germination et à l'établissement de lépinette rouge sont encore peu connues. Malgré qu'il y ait parfois une quantité appréciable de nouveaux semis à la suite d'une coupe partielle, ils disparaissent à court terme (Prévost et Charette 2015).

La présente étude vise à caractériser les conditions de germination et d'établissement de semis naturels dépinette rouge dans des trouées sylvicoles de différentes tailles (petites, moyennes et grandes). Nos hypothèses sont les suivantes : (1) la quantité de semis et leur survie seront plus élevées dans les petites trouées, (2) la taille des trouées et leurs conditions influenceront la répartition et le succès détablissement des semis en fonction de variables importantes du milieu (p. ex. le type de lit de germination), (3) la croissance des semis survivants sera optimale dans les grandes trouées, et (4) le développement des semis sera très lent, même dans des conditions favorables d'ouverture partielle du couvert forestier.

\section{Méthodes}

\section{Aire d'étude}

Létude a été conduite dans une bétulaie jaune résineuse des Appalaches $\left(46^{\circ} 50^{\prime} \mathrm{N}, 70^{\circ} 33^{\prime} \mathrm{W}\right.$, altitude : $\left.400 \mathrm{~m}\right)$, près de la municipalité d'Armagh, à environ $50 \mathrm{~km}$ à l'est de la ville de
Québec, Canada. La topographie y est plane et le sol est un podzol humo-ferrique. Le climat est de type continental subpolaire et subhumide. La température et la précipitation annuelles moyennes sont, respectivement, de $3,9^{\circ} \mathrm{C}\left(-12,8^{\circ} \mathrm{C}\right.$ en janvier et $18,5^{\circ} \mathrm{C}$ en juillet) et $1167 \mathrm{~mm}$, dont $23 \%$ sous forme de neige (MDDELCC 2015). Avant la coupe, le peuplement de l'aire d'étude avait une surface terrière marchande (ST) de $31 \mathrm{~m}^{2} \mathrm{ha}^{-1}$, composée à $37 \%$ de bouleau jaune (Betula alleghaniensis Britt.), $23 \%$ d'épinette rouge, $22 \%$ de sapin baumier, $15 \%$ d'érable rouge (Acer rubrum L.) et $2 \%$ d'essences compagnes représentées par le bouleau à papier (Betula papyrifera Marsh.) et lérable de Pennsylvanie (Acer pensylvanicum L.). Les structures d'âge et de diamètre du peuplement étaient irrégulières inéquiennes et les arbres dominants avaient une hauteur moyenne de $20 \mathrm{~m}$. La régénération préétablie était composée dépinette rouge et de sapin baumier.

\section{Dispositif expérimental}

Un dispositif sylvicole établi en 2003 (Prévost et Charette 2015) a été utilisé pour mener la présente étude. Chacune des trois répétitions utilisées (blocs) contenait quatre traitements de coupe, appliqués sur des unités expérimentales (UE) mesurant $80 \times 80 \mathrm{~m}$ : (i) une coupe partielle uniforme par pieds d'arbres, créant des ouvertures de taille $<100 \mathrm{~m}^{2}$ (petites trouées) et prélevant au total $42 \%$ de la ST, (ii) un traitement hybride de coupe partielle uniforme par pieds d'arbres et de coupe par petits groupes d'arbres, créant trois à cinq ouvertures de forme irrégulière et de taille variant de 100 à $300 \mathrm{~m}^{2}$ (moyennes trouées) et prélevant au total $35 \%$ de la ST, (iii) un traitement hybride de coupe partielle uniforme par pieds d'arbres et de coupe par trouée systématique, créant une ouverture circulaire $\left(30 \mathrm{~m}\right.$ de diamètre) de $700 \mathrm{~m}^{2}$ (grande trouée) et prélevant $40 \%$ de la ST dans le pourtour de la trouée, et (iv) un témoin naturel, donc sans intervention. Sur le plan sylvicole, lapproche hybride utilisée permettait entre autres, par le prélèvement de la mortalité potentielle entre les trouées, d'augmenter la vigueur du peuplement résiduel (Nyland 2002) et de rentabiliser l'opération. Afin de maintenir une source adéquate de graines pour régénérer lépinette rouge, presque tous les arbres semenciers de cette espèce dont le diamètre à hauteur de poitrine (DHP), mesuré à $1,3 \mathrm{~m}$ du sol, était supérieur ou égal à $25 \mathrm{~cm}$ ont été épargnés de la coupe. Ces arbres étaient uniformément répartis dans le peuplement. Leur densité et DHP moyens étaient respectivement 39 tiges ha- ${ }^{-1}$ et $34 \mathrm{~cm}$.

\section{Disponibilité de la lumière dans les trouées}

Durant les six premières années à la suite de la coupe, Prévost et Charette (2015) ont mesuré, de juin à octobre, la disponibilité de la lumière dans un des quatre blocs du dispositif expérimental. Pour ce faire, ils ont installé des pyranomètres (SPLITE, Kipp \& Zonen, Delft, Pays-Bas) à 1,3 m du sol et les ont reliés à un système automatique d'acquisition de données (CR10X, Campbell Scientific Canada, Edmonton, Alberta). Dans le témoin et la coupe partielle par pieds d'arbres (petites trouées), deux et trois pyranomètres ont respectivement été placés près du centre des UE. Dans les moyennes et grandes trouées, cinq pyranomètres ont été placés : un au centre de la trouée et quatre en périphérie, à $5 \mathrm{~m}$ de la bordure et disposés selon les points cardinaux (N, S, E et $\mathrm{O})$. 


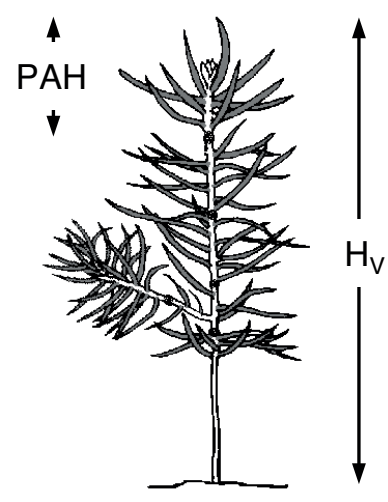

$\mathrm{NBV}=1$

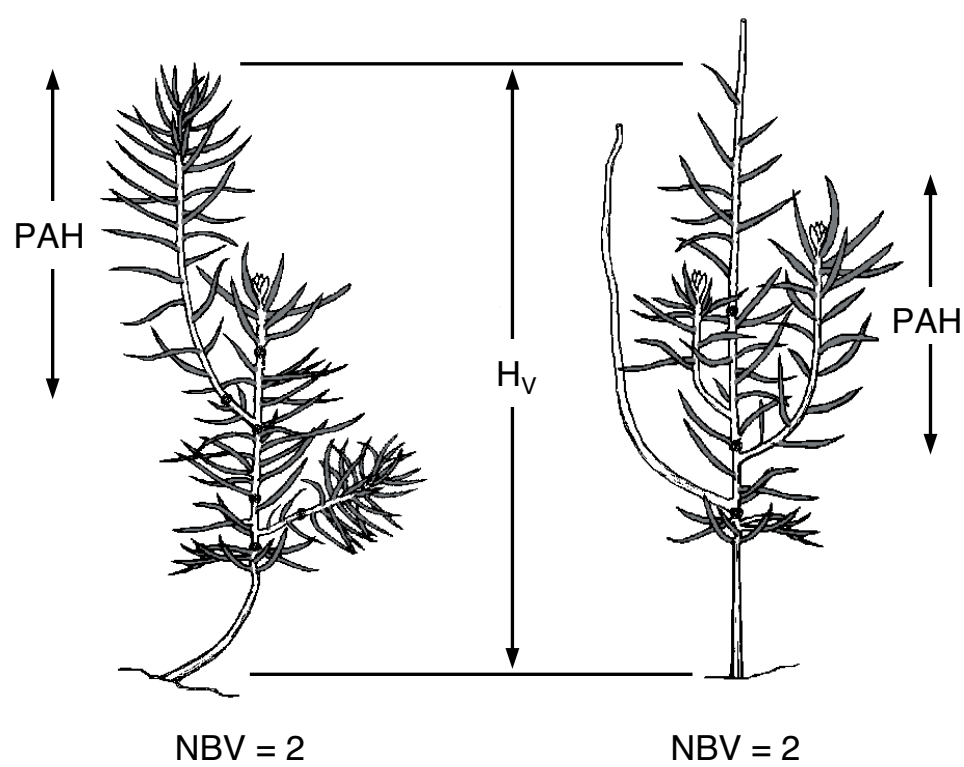

Fig. 1. Exemples de mesures de la pousse annuelle en hauteur $(\mathrm{PAH})$, de la hauteur vivante $\left(\mathrm{H}_{v}\right)$ et du nombre de branches vivantes (NBV) sur les semis d'épinette rouge.

\section{Échantillonnage et mesures}

Pour les besoins de cette étude, les observations dans chaque UE ont été faites dans une sous-parcelle centrale de $40 \mathrm{~m} \times$ $40 \mathrm{~m}$, composée d'une matrice variable de trouées et de peuplement éclairci créée par les différents traitements. Le recensement de nouveaux semis a été fait durant les trois premières années après la coupe, ce qui correspond au laps de temps où les conditions créées par l'ouverture du couvert et la perturbation du sol sont davantage favorables à l'installation de semis (Prévost et Pothier 2003). Au-delà de cette période et jusquà 10 ans après la coupe, le recrutement dépinette que nous avons observé a été en effet négligeable puisquaucune autre perturbation notable nest survenue dans les limites du dispositif expérimental. Ainsi, durant les trois premières années après la coupe, nous avons annuellement localisé chaque nouveau semis d'épinette rouge (tôt au printemps, avant la saison de végétation, et tard à lautomne, avant la première neige) en l'identifiant à l'aide d'une fiche métallique. Pour faciliter cette tâche, nous avons quadrillé la sous-parcelle en 16 surfaces déchantillonnage de $10 \mathrm{~m} \times 10 \mathrm{~m}$.

Pour chaque semis, la microtopographie a été classée selon trois catégories : (1) surface plane (plat), (2) butte, (3) creux. Une dénivellation d'au moins $15 \mathrm{~cm}$ par rapport au niveau du sol environnant servait de critère pour définir si le semis était situé dans un creux ou sur une butte. Le lit de germination a été caractérisé selon quatre catégories : (1) sol minéral exposé en surface (ou mélangé avec de l'humus), (2) mousse, (3) humus exposé, (4) bois en décomposition. La présence de broutement a été notée, peu importe son emplacement sur le semis, et toutes les espèces concurrentes ont été inventoriées (identification, hauteur) dans un rayon de $30 \mathrm{~cm}$ autour du semis et jusquà une hauteur de $1,5 \mathrm{~m}$.

Pour étudier leur développement, nous avons mesuré la pousse annuelle en hauteur (PAH) de chaque semis durant les dix premières années après la coupe. La PAH correspondait à la longueur de la pousse annuelle en hauteur la plus haute, soit la plus susceptible d'assurer le prolongement vertical du tronc (Fig. 1). Cette mesure a été prise en raison du manque de régularité architecturale et de la fréquence élevée de bris qui affectent la morphologie des jeunes semis (p. ex. : écrasement par la litière, broutement). Puisque la $\mathrm{PAH}$ ne correspondait pas toujours au gain annuel en hauteur, lévolution en hauteur a été déterminée en mesurant annuellement la hauteur vivante $\left(\mathrm{H}_{\mathrm{v}}\right)$, soit la longueur verticale entre le plus haut niveau du sol et la feuille vivante la plus haute (Fig. 1).

Tous les semis ont également fait l'objet d'un suivi annuel pour mesurer le broutement et la végétation concurrente tel que décrit précédemment. Dix ans après la coupe, nous avons de plus mesuré le diamètre au collet $\left(\right.$ Diam $\left._{c}\right)$ de chaque semis et le nombre de branches vivantes (NBV), soit la quantité d'axes primaires sur la tige principale (Fig. 1).

\section{Traitement des données et analyses statistiques}

À la fin de l'année 3, nous avons calculé la densité initiale $\left(D_{\text {ini }}\right)$, soit le nombre de semis dépinette rouge inventoriés durant les trois premières années après la coupe (période de recensement). De même, à la fin de l'année 10, nous avons calculé la densité finale $\left(\mathrm{D}_{\text {fin }}\right)$, ce qui nous a permis de calculer le taux de survie décennal $\left(\mathrm{TS}_{10}\right)$, qui correspondait au nombre de semis encore vivants divisé par la $\mathrm{D}_{\mathrm{ini}}$, exprimé en $\%$. Ce calcul simple prenait donc pour acquis qu'il ny avait pas eu d'installation significative de nouveaux semis dépinette après trois ans, tel qu’observé sur le terrain.

Les données de $\mathrm{D}_{\text {ini, }}, \mathrm{D}_{\text {fin' }}, \mathrm{TS}_{10}$, Diam $_{\mathcal{c}^{\prime}}$ NBV, PAH et $\mathrm{H}_{\mathrm{v}}$ ont été soumises à une analyse de variance en utilisant la procédure MIXED du logiciel SAS (version 9.3). La PAH et la $\mathrm{H}_{\mathrm{v}}$ ont été analysées en mesures répétées dans le temps sur les mêmes individus. Des tests de comparaisons multiples ont été 
Tableau 1. Structure des analyses de variance et probabilités $(p>F)$ associées aux effets de la taille des trouées sylvicoles (Témoin : sans trouée, Petite : $<100 \mathrm{~m}^{2}$, Moyenne : 100-300 $\mathrm{m}^{2}$ de forme irrégulière, Grande : $700 \mathrm{~m}^{2}$ circulaire) sur la densité initiale $\left(D_{\text {ini, }}\right.$, année 3$)$ et finale $\left(D_{\text {fin }}\right.$, année 10$)$ de semis d'épinette rouge, leur taux de survie décennal $\left(T S_{10}\right)$, leur diamètre au collet (Diam, ${ }_{c}$, année 10) et leur nombre de branches vivantes (NBV, année 10).

\begin{tabular}{|c|c|c|c|c|c|c|c|}
\hline \multirow{2}{*}{$\begin{array}{l}\text { Source } \\
\text { de variation }\end{array}$} & \multirow[b]{2}{*}{$\operatorname{dln}^{1}$} & \multirow[b]{2}{*}{ dld $^{2}$} & \multicolumn{5}{|c|}{$p$} \\
\hline & & & $\mathrm{D}_{\text {ini }}$ & $\mathrm{D}_{\text {fin }}$ & $\mathrm{TS}_{10}$ & $\operatorname{Diam}_{c}$ & NBV \\
\hline Taille des trouées & 3 & 6 & 0,009 & 0,023 & $<0,001$ & $<0,001$ & 0,004 \\
\hline Témoin $v s$ petite & 1 & 6 & 0,010 & 0,017 & 0,002 & 0,016 & 0,102 \\
\hline Témoin vs moyenne & 1 & 6 & 0,830 & 0,040 & $<0,001$ & 0,035 & 0,230 \\
\hline Témoin $v s$ grande & 1 & 6 & 0,877 & 0,561 & 0,107 & 0,001 & 0,003 \\
\hline Petite $v s$ moyenne & 1 & 6 & 0,023 & 0,037 & 0,167 & 0,861 & 0,880 \\
\hline Petite $v s$ grande & 1 & 6 & 0,020 & 0,026 & 0,027 & 0,009 & 0,028 \\
\hline Moyenne vs grande & 1 & 6 & 0,999 & 0,048 & 0,002 & 0,004 & 0,014 \\
\hline
\end{tabular}

${ }^{1} \mathrm{dln}$ : degrés de liberté au numérateur.

${ }^{2} \mathrm{dld}$ : degrés de liberté au dénominateur (exemple pour la $\mathrm{D}_{\mathrm{ini}}$ ).

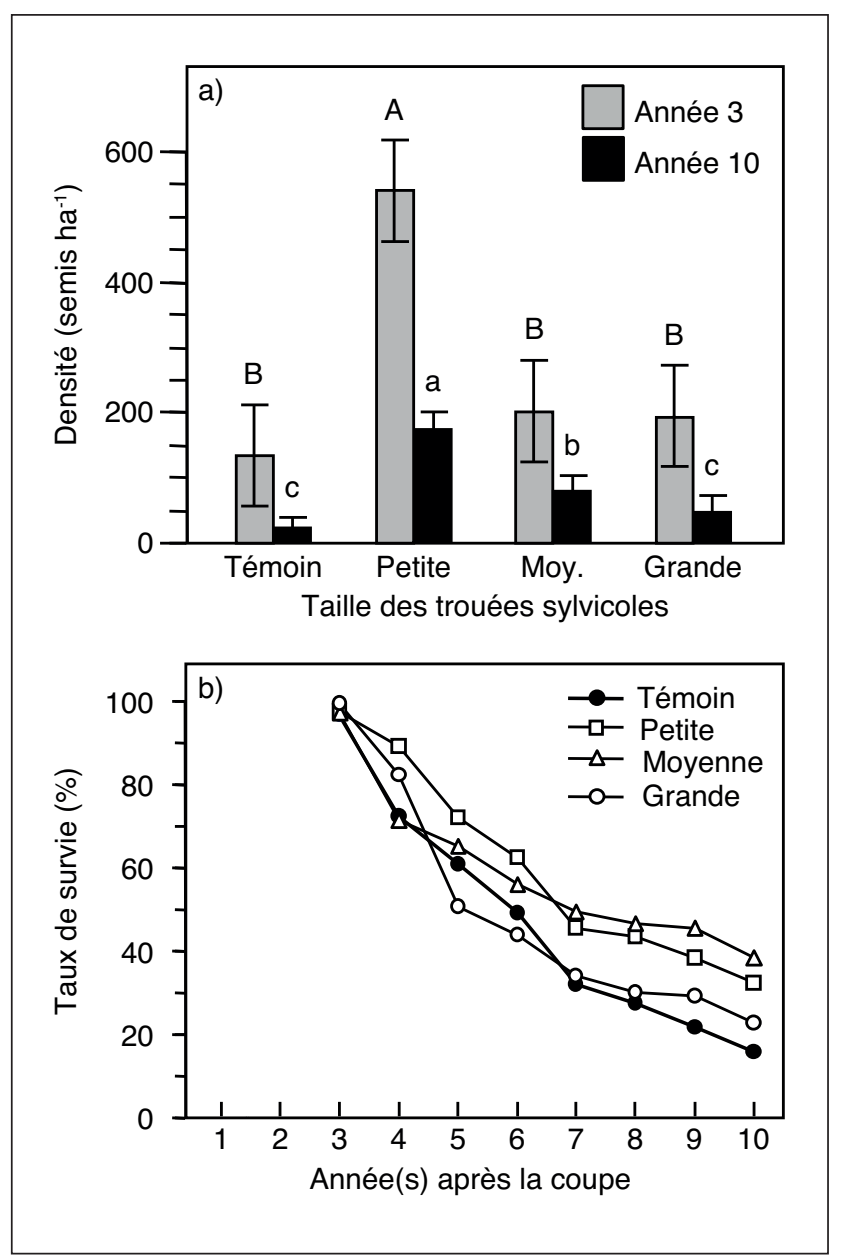

Fig. 2. a) Effet de la taille des trouées sylvicoles (définies au Tableau 1) sur la densité initiale (année 3) et finale (année 10) de semis d'épinette rouge. Pour une même année, les colonnes de l'histogramme ayant la même lettre ont des valeurs qui ne sont pas statistiquement différentes. Les barres verticales montrent l'erreur-type. b) Effets de la taille des trouées sylvicoles sur le taux de survie annuel des semis. L'erreur-type maximale est de $\pm 2 \%$. menés (option SIMULATE) pour vérifier l'effet de la taille des trouées. Des contrastes polynomiaux ont été utilisés afin d'interpréter les interactions significatives entre la taille des trouées et le temps.

La pression de broutement a été incluse dans le modèle en tant que variable qualitative à quatre modalités : (1) le semis n'a pas été brouté, (2) il a été brouté lors d'une année, (3) deux années, (4) trois années et plus. De même, la végétation concurrente dominante a été incluse selon cinq modalités : (1) le semis nétait accompagné d'aucune végétation, (2) il était accompagné d'essences feuillues, (3) de résineux, (4) de fougères, (5) de framboisier. La hauteur et la densité ont servi de critères pour déterminer la végétation concurrente dominante de chaque semis. Les données de répartition des semis selon la microtopographie, le lit de germination, loccurrence de broutement et la végétation concurrente ont été soumises séparément à des analyses de variance afin de vérifier l'effet de la taille des trouées. Des analyses séparées ont été nécessaires puisque la distribution des données ne permettait pas de tester adéquatement les interactions entre les variables du milieu.

Pour étudier les liens entre la survie, le développement et les variables du milieu, le $\mathrm{TS}_{10}$ et la $\mathrm{PAH}$ ont été recalculées par type de microtopographie, lit de germination, occurrence de broutement et végétation concurrente. Nous avons ensuite effectué, pour chaque taille de trouées, des analyses de variance faisant intervenir chaque variable du milieu.

Pour les tests statistiques, un seuil de signification de 0,05 a été retenu. L'homogénéité de la variance a été vérifiée graphiquement. Des transformations mathématiques ont été appliquées aux données montrant de l'hétéroscédasticité (logarithme naturel pour la $\mathrm{PAH})$, lesquelles ont été ramenées à leur forme originale pour la présentation des résultats.

\section{Résultats}

Disponibilité de la lumière dans les trouées

Durant les six premières années, la disponibilité moyenne de la lumière a augmenté avec l'ouverture du couvert forestier. Elle était de 1,5, 3,9, 4,9 et 8,9 $\mathrm{MJ} \mathrm{m}^{-2} \mathrm{j}^{-1}$, respectivement dans le témoin et dans les petites, moyennes et grandes trouées. 
Densité et survie selon la taille des trouées

La $\mathrm{D}_{\text {ini }}$ de semis deépinette des petites trouées a été plus élevée (534 semis ha-1) que celles du témoin (135) et des plus grandes trouées (196-198) ( $p \leq 0,023$, Tableau 1, Fig. 2a). La survie a diminué rapidement durant les années 4 à 6 , et lentement ensuite (Fig. 2b). La taille des trouées a eu un effet sur le $\mathrm{TS}_{10}$. À peine $23 \%$ des semis ont survécu dans les grandes trouées, ce qui n'a pas été significativement différent du $\mathrm{TS}_{10}$ de $16 \%$ du témoin ( $p=0,107$, Tableau 1$)$. Les $\mathrm{TS}_{10}$ ont été plus élevés dans les petites (32\%) et moyennes (38\%) trouées que dans les grandes ( $p \leq 0,027$, Fig. 2b). Ainsi, à l'année 10, la $\mathrm{D}_{\text {fin }}$ était supérieure dans les petites trouées (173 semis ha-1), intermédiaire dans les moyennes trouées (76) et inférieure dans les grandes trouées (45) et le témoin (21) ( $p \leq 0,037$, Tableau 1, Fig. 2a).

\section{Microtopographie}

Le nombre de semis dans des creux a été nul. À l'année 3, la proportion de semis occupant les buttes diminuait avec louverture plus prononcée du couvert forestier (Fig. 3a). Elle était plus grande dans le témoin que dans les trouées $(p \leq 0,001$, Tableau $2 \mathrm{a}$ ), et plus grande dans les petites trouées que dans les grandes trouées $(p=0,010)$. À l'inverse, la proportion de semis occupant le sol plat augmentait avec l'ouverture du couvert. Elle était plus faible dans le témoin que dans les moyennes et grandes trouées $(p \leq 0,005)$, et plus faible dans les petites et moyennes trouées que dans les grandes $(p \leq 0,008)$. En général, le patron observé selon l'ouverture du couvert s'est maintenu à l’année 10. Cependant, la meilleure survie sur les buttes ( $p \leq 0,044$, Tableau 3a, Fig. 4a) a fait que plus de $60 \%$ des semis survivants s'y retrouvaient (Fig. 3a). Le témoin se démarquait toujours des trouées $(p<0,001$, non présenté) avec $95 \%$ de ses semis sur des buttes.

\section{Lit de germination}

À l'année 3, la proportion de semis sur du bois en décomposition ou de la mousse diminuait avec l'augmentation de la taille des trouées (Fig. 3b). La proportion de semis sur du bois était plus grande dans le témoin que dans les trouées ( $p \leq 0,041$, Tableau 2b), et plus grande dans les petites trouées que dans les grandes $(p=0,027)$. La proportion de semis sur de la mousse était plus grande dans le témoin que dans les moyennes et grandes trouées $(p \leq 0,001)$, et plus grande dans les petites trouées que dans les plus grandes $(p \leq 0,017)$. À l'inverse, la proportion de semis occupant l'humus à l'année 3 augmentait avec l'ouverture du couvert (Fig. 3b). Elle était beaucoup plus faible dans le témoin que dans les trouées $(p \leq 0,002)$, et plus faible dans les petites trouées que dans les plus grandes $(p \leq 0,004)$. Le sol minéral a été colonisé uniquement dans les grandes trouées, et ce, avec une faible proportion de semis (10\%, année 3) dont la survie et la quantité décennale restante ont été négligeables (Figs. 3b et $4 \mathrm{~b}$ ). La dynamique de survie a changé le patron observé selon l'ouverture du couvert à l'année 10 (Fig. 3b). Dans les grandes trouées, la survie nettement plus élevée sur le bois en décomposition ( $p \leq 0,038$, Tableau 3b, Fig. $4 \mathrm{~b}$ ) a fait que $60 \%$ des semis survivants se retrouvaient alors sur ce substrat plutôt que sur l'humus (année 3).

\section{Occurrence de broutement}

La proportion de semis exempts de broutement au cours des 10 ans d’observation a été plus élevée dans le témoin (70 \%)

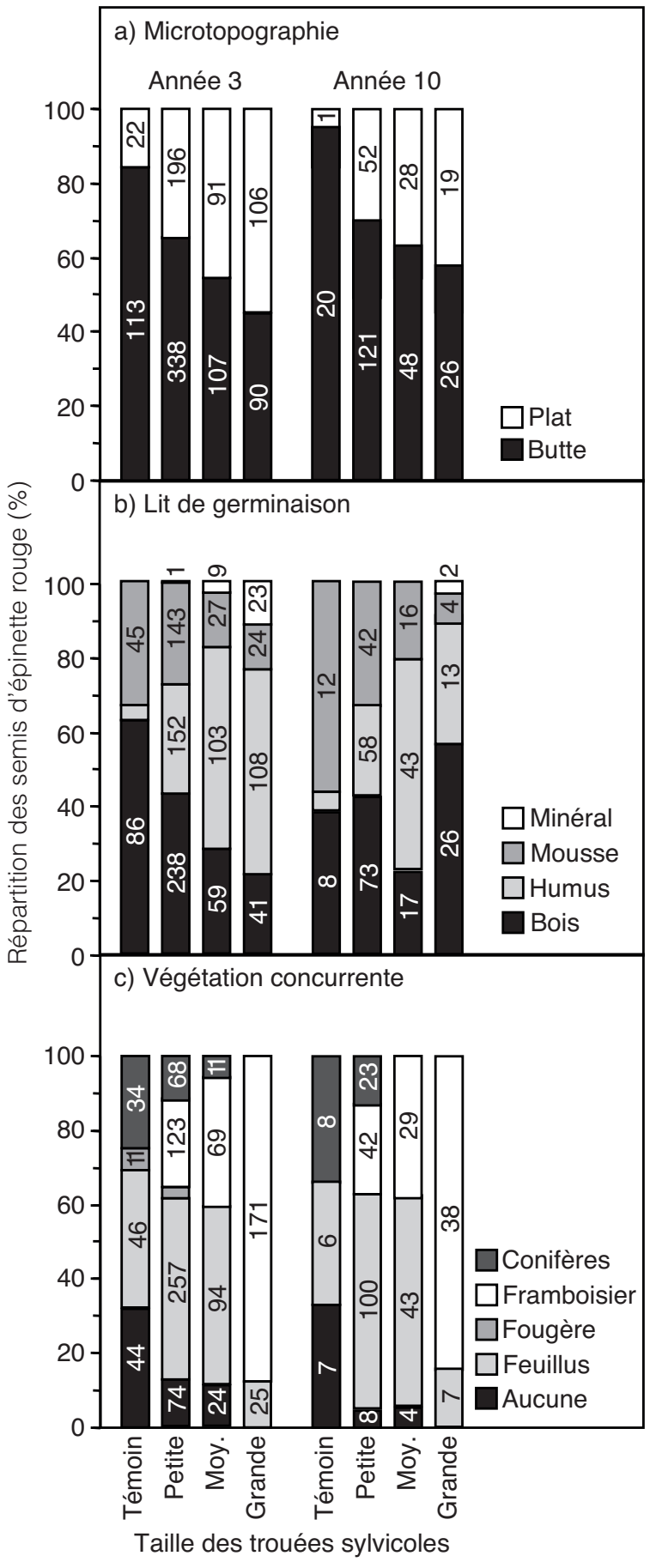

Fig. 3. Effets de la taille des trouées sylvicoles (définies au Tableau 1) sur la répartition des semis d'épinette rouge en fonction a) de la microtopographie, b) du lit de germination et c) de la végétation concurrente dominante. L'erreur-type maximale est de $\pm 3 \%$ pour la microtopographie, $\pm 4 \%$ pour le lit de germination et $\pm 6 \%$ pour la végétation concurrente. Les nombres inscrits sur les colonnes d'histogramme indiquent la densité (semis ha-1 ${ }^{-1}$. En raison du manque d'espace, les densités suivantes n'ont pas été inscrites sur la figure : b) Humus dans le témoin = 4 (année 3 ) et 1 (année10), c) Fougère dans les petites trouées $=12$ (année 3 ). 
Tableau 2. Structure des analyses de variance et probabilités $(p>F)$ associées aux effets de la taille des trouées sylvicoles (définies au Tableau 1) sur la répartition des semis d'épinette rouge selon les variables du milieu, soit la microtopographie (année 3; année 10 non présentée), le lit de germination (année 3; 10 non présentée), l'occurrence de broutement (sur 10 ans) et la végétation concurrente (année 3; 10 non présentée].

a) Microtopographie

\begin{tabular}{lccrrr} 
Source de variation & $\mathbf{d l n}^{\mathbf{1}}$ & dld $^{\mathbf{2}}$ & Butte & Plat \\
\hline Taille des trouées & 3 & 8 & $<0,001$ & $<0,001$ \\
Témoin $v$ s petite & 1 & 8 & 0,001 & 0,071 \\
Témoin $v$ s moyenne & 1 & 8 & $<0,001$ & 0,005 \\
Témoin $v$ s grande & 1 & 8 & $<0,001$ & $<0,001$ \\
Petite $v s$ moyenne & 1 & 8 & 0,134 & 0,163 \\
Petite $v s$ grande & 1 & 8 & 0,010 & $<0,001$ \\
Moyenne $v s$ grande & 1 & 8 & 0,295 & 0,008
\end{tabular}

b) Lit de germination

\begin{tabular}{rrrr}
\hline Bois & Mousse & Humus & Minéral \\
\hline 0,001 & $<0,001$ & $<0,001$ & - \\
0,041 & 0,244 & 0,002 & - \\
0,002 & 0,001 & $<0,001$ & - \\
0,001 & $<0,001$ & $<0,001$ & - \\
0,124 & 0,017 & 0,004 & - \\
0,027 & 0,003 & 0,002 & - \\
0,666 & 0,577 & 0,954 & -
\end{tabular}

c) Broutement (occurrence)

$\begin{array}{lll}\text { Taille des trouées } & 3 & 8 \\ \text { Témoin } v s \text { petite } & 1 & 8 \\ \text { Témoin } v s \text { moyenne } & 1 & 8 \\ \text { Témoin } v s \text { grande } & 1 & 8 \\ \text { Petite } v s \text { moyenne } & 1 & 8 \\ \text { Petite } v s \text { grande } & 1 & 8 \\ \text { Moyenne } v s \text { grande } & 1 & 8\end{array}$

\begin{tabular}{cccc}
\hline Aucun & $\mathbf{1}$ an & $\mathbf{2}$ ans & $\mathbf{3}$ et + \\
\hline 0,016 & 0,470 & 0,281 & 0,464 \\
0,017 & 0,718 & 0,743 & 0,627 \\
0,032 & 0,940 & 0,234 & 0,677 \\
0,050 & 0,987 & 0,829 & 0,441 \\
0,932 & 0,417 & 0,655 & 1,000 \\
0,746 & 0,886 & 0,998 & 0,984 \\
0,973 & 0,808 & 0,564 & 0,971
\end{tabular}

d) Végétation concurrente dominante

\begin{tabular}{rrrr}
\hline Aucune & Feuillus & $\begin{array}{r}\text { Fram- } \\
\text { boisier }\end{array}$ & Conifères \\
\hline 0,025 & 0,001 & 0,009 & 0,011 \\
0,035 & 0,235 & - & 0,048 \\
0,029 & 0,295 & - & 0,009 \\
- & 0,007 & - & - \\
0,962 & 0,998 & 0,757 & 0,370 \\
- & 0,001 & 0,012 & - \\
- & 0,001 & 0,027 & - \\
\hline
\end{tabular}

${ }^{1} \mathrm{dln}$ : degrés de liberté au numérateur (exemple pour la première variable).

${ }^{2}$ dld : degrés de liberté au dénominateur (exemple pour la première variable). que dans les trouées $(50 \%)(p \leq 0,050$, Tableau 2c). La survie ne semble pas avoir été affectée par loccurrence de broutement ( $p \geq 0,118$, Tableau 3c).

\section{Végétation concurrente dominante}

La proportion de semis exempts de végétation concurrente au cours des 10 ans de suivi a été plus élevée dans le témoin que dans les petites et moyennes trouées $(p \leq 0,035$, Tableau 2d, Fig. 3c). Aucun semis n'a été exempt de végétation concurrente dans les grandes trouées, où le framboisier sest avéré être le concurrent dominant de presque tous les semis. Par ailleurs, la proportion de semis aux prises avec le framboisier a été comparable dans les petites et moyennes trouées, mais elle a été plus élevée dans les grandes trouées $(p \leq 0,027)$. De même, la proportion de semis étant en compétition avec des feuillus a été similaire dans le témoin et dans les petites et moyennes trouées, mais beaucoup plus faible dans les grandes trouées $(p \leq 0,007)$. Quant aux conifères, cest dans le témoin qu'ils ont concurrencé une proportion notable de semis $(p \leq 0,048)$. Comme la nature de la végétation na pas significativement affecté la survie (Fig. 4c), le patron de répartition des semis a peu changé à l'année 10 (Fig. 3c). Néanmoins, dans les petites et moyennes trouées, les semis avec aucune concurrence ont occupé significativement moins de place à lannée 10 quà l'année $3(p \leq 0,032)$. Cela a été causé par un $\mathrm{TS}_{10}$ réduit de ces semis par rapport à ceux accompagnés de végétation concurrente $(p \leq 0,044$, Tableau $3 \mathrm{~d}$, Fig. 4c).

\section{Variables de croissance selon la taille} des trouées

Après 10 ans, le Diam $c$ et le NBV étaient liés à la taille des trouées. Le Diam $_{c}$ était plus grand dans les trouées (24 à $40 \mathrm{~mm})$ que dans le témoin $(14 \mathrm{~mm})(p \leq 0,035$, Tableau 1). Quant au NBV, il était plus élevé dans les grandes trouées (12) que dans les plus petites (6-7) et le témoin (3) ( $p \leq$ $0,028)$.

L'interaction entre la taille des trouées et le temps a eu un effet sur la PAH ( $p<0,001$, Tableau 4$)$. La PAH a généralement été plus grande dans les trouées $\left(2\right.$ à $\left.4 \mathrm{~cm} \mathrm{an}^{-1}\right)$ que dans le témoin $\left(1 \mathrm{~cm} \mathrm{an}^{-1}\right)$, et plus grande 
Tableau 3. Structure des analyses de variance et probabilités ( $p>F$ ) associées aux effets des variables du milieu sur le taux de survie décennal $\left(\mathrm{TS}_{10}\right.$ ) des semis d'épinette rouge, selon la taille des trouées sylvicoles (définies au Tableau 1).

\begin{tabular}{|c|c|c|c|c|c|c|c|c|c|c|c|c|}
\hline \multirow[b]{3}{*}{ Source de variation } & \multicolumn{12}{|c|}{ Taille des trouées sylvicoles } \\
\hline & \multicolumn{3}{|c|}{ Témoin } & \multicolumn{3}{|c|}{ Petite } & \multicolumn{3}{|c|}{ Moyenne } & \multicolumn{3}{|c|}{ Grande } \\
\hline & $d \ln ^{1}$ & dld $^{2}$ & $p$ & dln & dld & $p$ & dln & dld & $p$ & dln & dld & $p$ \\
\hline \multicolumn{13}{|l|}{ a) Microtopographie } \\
\hline Butte vs plat & 1 & 2,0 & 0,015 & 1 & 2,3 & 0,043 & 1 & 2,0 & 0,039 & 1 & 2,0 & 0,044 \\
\hline \multicolumn{13}{|l|}{ b) Lit de germination } \\
\hline Bois vs humus & 1 & 4,0 & 0,590 & 1 & 6,0 & 0,682 & 1 & 4,0 & 0,646 & 1 & 3,0 & 0,038 \\
\hline Bois $v s$ mousse & 1 & 4,0 & 0,304 & 1 & 6,0 & 0,984 & 1 & 4,0 & 0,235 & 1 & 2,9 & 0,031 \\
\hline Bois $v s$ minéral & - & - & - & - & - & - & - & - & - & 1 & 3,0 & 0,016 \\
\hline Humus $v s$ mousse & 1 & 4,0 & 0,891 & 1 & 6,0 & 0,577 & 1 & 4,0 & 0,579 & 1 & 3,0 & 0,892 \\
\hline Humus $v s$ minéral & - & - & - & - & - & - & - & - & - & 1 & 2,9 & 0,623 \\
\hline Mousse $v s$ minéral & - & - & - & - & - & - & - & - & - & 1 & 3,0 & 0,457 \\
\hline \multicolumn{13}{|l|}{ c) Broutement (occurrence) } \\
\hline Aucun $v s 1$ an & 1 & 4,5 & 0,997 & 1 & 8,0 & 0,999 & 1 & 5,4 & 0,933 & 1 & 6,0 & 0,999 \\
\hline Aucun $v s 2$ ans & 1 & 4,9 & 0,618 & 1 & 8,0 & 0,627 & 1 & 5,4 & 0,964 & 1 & 6,0 & 0,990 \\
\hline Aucun vs 3 ans+ & 1 & 4,9 & 0,933 & 1 & 8,0 & 0,474 & 1 & 5,8 & 0,190 & 1 & 6,0 & 0,998 \\
\hline 1 an $v s 2$ ans & 1 & 4,9 & 0,707 & 1 & 8,0 & 0,580 & 1 & 5,3 & 0,728 & 1 & 6,0 & 0,972 \\
\hline 1 an $v s 3$ ans+ & 1 & 4,9 & 0,870 & 1 & 8,0 & 0,430 & 1 & 5,7 & 0,342 & 1 & 6,0 & 0,987 \\
\hline 2 ans vs 3 ans + & 1 & 4,5 & 0,409 & 1 & 8,0 & 0,993 & 1 & 5,7 & 0,118 & 1 & 6,0 & 1,000 \\
\hline \multicolumn{13}{|l|}{ d) Végétation concurrente } \\
\hline Aucune $v s$ feuillus & 1 & 6,0 & 0,788 & 1 & 6,0 & 0,018 & 1 & 4,0 & 0,041 & - & - & - \\
\hline Aucune $v s$ conifères & 1 & 6,0 & 0,594 & 1 & 6,0 & 0,036 & - & - & - & - & - & - \\
\hline Aucune $v s$ framboisier & - & - & - & 1 & 6,0 & 0,039 & 1 & 4,0 & 0,044 & - & - & - \\
\hline Feuillus $v s$ conifères & 1 & 6,0 & 0,290 & 1 & 6,0 & 0,945 & - & - & - & - & - & - \\
\hline Feuillus $v s$ framboisier & - & - & - & 1 & 6,0 & 0,903 & 1 & 4,0 & 0,903 & 1 & 3,0 & 0,768 \\
\hline Framboisier $v s$ conifères & - & - & - & 1 & 6,0 & 0,999 & - & - & - & - & - & - \\
\hline
\end{tabular}

${ }^{1} \mathrm{dln}$ : degrés de liberté au numérateur.

${ }^{2} \mathrm{dld}$ : degrés de liberté au dénominateur.

dans les grandes trouées que les plus petites (Fig. 5a). L'interaction indique que lécart entre le témoin et les petites et moyennes trouées a diminué avec le temps $(p \leq 0,003)$, de sorte quaprès 10 ans, les semis des petites et moyennes trouées avaient une croissance en hauteur aussi faible que ceux du témoin (Fig. 5a). Au contraire, lécart de PAH entre les grandes trouées et le témoin $\left(+3 \mathrm{~cm} \mathrm{an}^{-1}\right)$ sest maintenu, et un écart plus important entre les grandes trouées et les autres est apparu $(p \leq 0,047)$.

Conséquemment à la $\mathrm{PAH}$, la taille des trouées et le temps ont eu un effet sur la $\mathrm{H}_{\mathrm{v}}$ des semis. En moyenne sur 10 ans, les semis étaient plus hauts dans les trouées $(13 \mathrm{~cm})$ que dans le témoin $(7 \mathrm{~cm})(p \leq 0,046$, Tableau 4 , Fig. $5 b)$, et plus hauts dans les grandes trouées $(16 \mathrm{~cm})$ que dans les plus petites $(11 \mathrm{~cm})(p \leq 0,029)$.

Variables du milieu influençant la croissance en hauteur La microtopographie a eu un effet significatif uniquement dans les moyennes trouées, où la PAH des semis a été supérieure de $1 \mathrm{~cm} \mathrm{an}^{-1}$ sur les surfaces planes par rapport aux buttes ( $p=0,017$, non présenté, Fig. $6 \mathrm{a})$. Dans les petites trouées, les lits de germination d'humus ou de mousse ont été plus favorables à la $\mathrm{PAH}$ des semis $\left(+1 \mathrm{~cm} \mathrm{an}^{-1}\right)$ que le bois en décomposition ( $p \leq 0,047$, Fig. $6 \mathrm{~b}$ ). Dans les moyennes trouées, l'humus sest avéré plus favorable $\left(+1 \mathrm{~cm} \mathrm{an}^{-1}\right)$ que les autres substrats $(p \leq 0,025)$, alors que dans les grandes trouées, c'est la mousse qui a été la plus bénéfique (jusquà $\left.+1 \mathrm{~cm} \mathrm{an}^{-1}, p \leq 0,036\right)$. Enfin, dans les petites et moyennes trouées, la PAH des semis accompagnés de feuillus ou de framboisier a été plus élevée $\left(+1 \mathrm{~cm} \mathrm{an}^{-1}\right)$ que celle des semis libres de concurrence ou accompagnés de conifères ( $p \leq 0,023$, Fig. 6c).

\section{Discussion}

\section{Effet des trouées sur la densité de nouveaux semis}

Les densités de semis, presque trois fois supérieures dans les petites trouées que dans les plus grandes (Fig. 2a), confirment notre première hypothèse d'un meilleur établissement dans les petites ouvertures $<100 \mathrm{~m}^{2}$. En outre, elles nous apparaissent révélatrices des conditions de lumière et de perturbation du sol favorables à la germination et à létablissement de l'espèce. Il semble donc qu'une mise en lumière modérée combinée à une perturbation légère du sol aient été optimales. Ce constat milite en faveur de la coupe partielle par pieds d'arbres (Dibble et al. 1999, Archambault et al. 2003, Prévost et Charette 2015), et va à l'encontre de l'hypothèse antérieure voulant que ce type de coupe puisse être trop léger pour créer des conditions propices à la régénération de l'épinette rouge (p. ex. Frank et Blum 1978). 


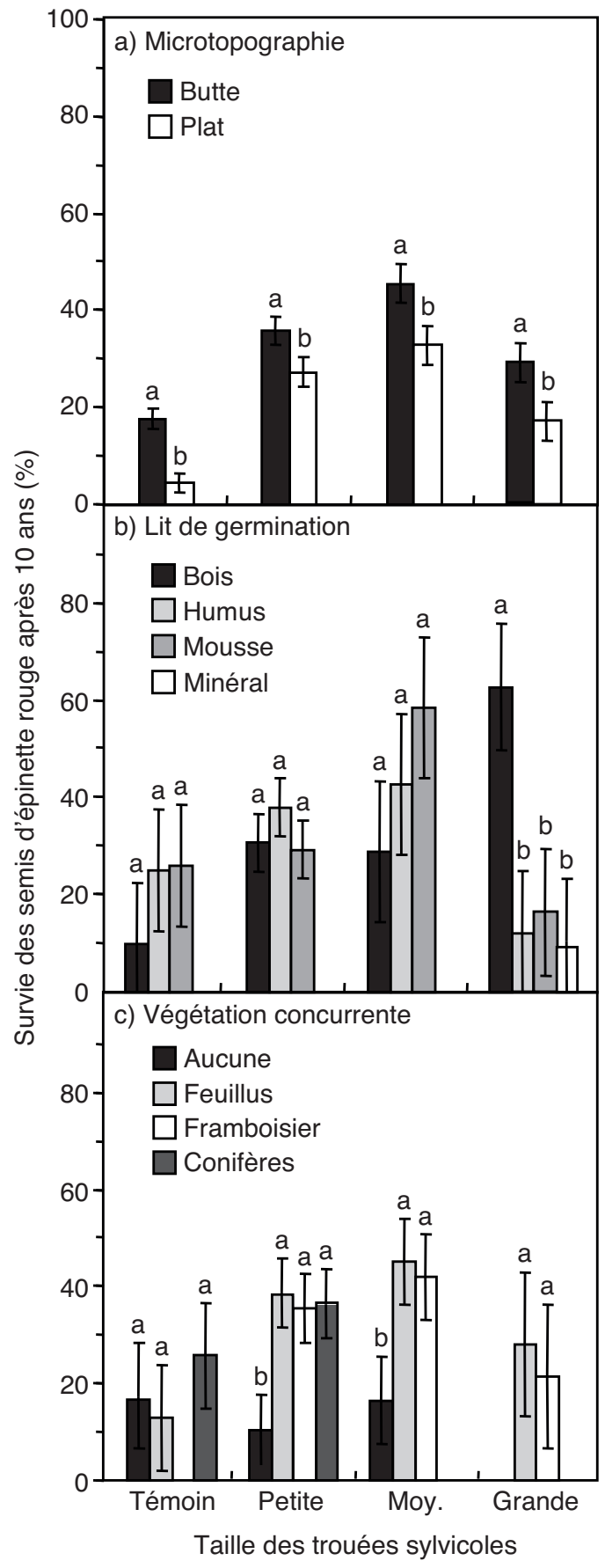

Fig. 4. Effets a) de la microtopographie, b) du lit de germination et c) de la végétation concurrente dominante sur le taux de survie décennal $\left(\mathrm{TS}_{10}\right.$ ) des semis d'épinette rouge, selon la taille des trouées (définies au Tableau 1). Pour une même taille de trouée, les colonnes de l'histogramme ayant la même lettre ont des valeurs qui ne sont pas statistiquement différentes. Les barres verticales montrent l'erreur-type.
Effet des trouées sur la germination et ses conditions (année 3) Notre seconde hypothèse, à l'effet que la taille des trouées sylvicoles avait une incidence sur la répartition des semis en fonction de variables du milieu, doit être vérifiée en gardant à l'esprit que la création des trouées a pu influencer à divers degrés ces variables. Par exemple, le recouvrement par le sol minéral a augmenté avec la perturbation du sol qui a été plus importante dans les moyennes et grandes trouées (Prévost et Charette 2015). Ainsi, la présence de nouveaux semis sur ce substrat pourraient refléter en partie la disponibilité de ce dernier plutôt que sa réceptivité pour lépinette. À cet effet, les travaux de Prévost et Charette (2015) qui ont étudié le recouvrement des lits de germination dans ce même dispositif expérimental nous aideront à interpréter les résultats de la présente étude.

Loccupation des buttes et surfaces planes par les semis a suivi les gradients de lumière et de perturbation du sol engendrés par l’augmentation de la taille des trouées (Fig. 3a). Dans les conditions ombragées et de sol non perturbé du témoin, les buttes ont été de loin plus propices à la germination que les surfaces planes grandement disponibles, possiblement en raison d'une température de substrat moins fraîche (Dwyer et Merriam 1981) et d'une accumulation moindre de litière (Ruel et al. 1988). Par contre, dans les grandes trouées de $700 \mathrm{~m}^{2}$, les surfaces planes ont dans la présente étude été initialement aussi prisées que les buttes, possiblement en raison d'une disponibilité en eau plus constante (Örlander et al. 1990) et de températures de l'air et du sol moins extrêmes.

La littérature souligne l'importance du bois en décomposition comme lit de germination privilégié de lépinette rouge (p. ex. Weaver et al. 2009), notamment parce qu'il a une bonne rétention en eau (Fraver et al. 2002). Notre étude fait ressortir la préférence de lépinette rouge pour ce type de substrat dans le témoin. En effet, même si ce substrat ne couvrait que $9 \%$ de la superficie sous couvert (Prévost et Charrette 2015), on y retrouvait plus de $60 \%$ des semis dépinette (Fig. 3b). Or, la proportion de semis sur du bois pourri a diminué avec l'ouverture du couvert, ce qui peut être en partie attribuable à une disponibilité moindre de ce substrat (5\% de la superficie dans les moyennes et grandes trouées) mais aussi à une teneur en humidité réduite de ce dernier. Avec davantage d'exposition au rayonnement solaire, il se peut que certains niveaux de décomposition du bois n'aient pas été en mesure de maintenir une humidité favorable à la germination (voir Robert et al. 2012). Ainsi, l'interaction entre le degré douverture du couvert et la présence de bois à différents stades de décomposition expliquerait mieux le succès de germination de lépinette rouge que la simple présence de ce substrat. Cette hypothèse serait à vérifier. Par ailleurs, nos résultats indiquent que l'humus serait davantage favorable à la germination de lespèce dans les moyennes et grandes trouées (Fig. 3b). En effet, même si l'humus n'occupait que 2 à $5 \%$ de la superficie de ces trouées (7-13\% pour le sol minéral et 4$5 \%$ pour le bois en décomposition, Prévost et Charette 2015), nous y avons retrouvé plus de $50 \%$ des semis.

La végétation concurrente a aussi été influencée par la taille des trouées, puisquelle dépend des conditions de lumière, d'humidité et de température du sol. Par exemple, le recouvrement par le framboisier a atteint 23 et $44 \%$, respectivement dans les moyennes et grandes trouées (Prévost et Charette 2015). Bien que $56 \%$ de la superficie des grandes 
Tableau 4. Structure des analyses de variance et probabilités ( $p>F$ ) associées aux effets de la taille des trouées (définie au Tableau 1) et du temps (années 2 à 10) sur la pousse annuelle en hauteur (PAH) et l'évolution annuelle de la hauteur vivante $\left(\mathrm{H}_{v}\right)$ des jeunes semis naturels d'épinette rouge.

\begin{tabular}{lccrr}
\hline $\begin{array}{l}\text { Source } \\
\text { de variation }\end{array}$ & $\mathbf{d l n}^{\mathbf{1}}$ & $\mathbf{d l d}^{\mathbf{2}}$ & PAH & $\mathbf{H}_{\mathbf{v}}$ \\
\hline Taille des trouées & 3 & 8,0 & 0,001 & 0,011 \\
Témoin $v s$ petite & 1 & 7,9 & 0,018 & 0,042 \\
Témoin $v$ moyenne & 1 & 8,0 & 0,041 & 0,046 \\
Témoin $v s$ grande & 1 & 8,0 & $<0,001$ & 0,001 \\
Petite $v s$ moyenne & 1 & 7,9 & 0,935 & 0,956 \\
Petite $v s$ grande & 1 & 8,1 & 0,040 & 0,029 \\
Moyenne $v s$ grande & 1 & 7,9 & 0,018 & 0,026 \\
Temps $(\mathrm{T})$ & 8 & 26,2 & $<0,001$ & $<0,001$ \\
Taille des trouées $\times \mathrm{T}$ & 24 & 47,4 & $<0,001$ & 0,144 \\
Témoin $v s$ petite $\times \mathrm{T}$ & 1 & 44,6 & 0,003 & - \\
Témoin $v s$ moyenne $\times \mathrm{T}$ & 1 & 61,5 & $<0,001$ & - \\
Témoin $v s$ grande $\times \mathrm{T}$ & 1 & 55,9 & 0,249 & - \\
Petite $v s$ moyenne $\times \mathrm{T}$ & 1 & 44,2 & 0,010 & - \\
Petite $v s$ grande $\times \mathrm{T}$ & 1 & 62,3 & 0,001 & - \\
Moyenne $v s$ grande $\times \mathrm{T}$ & 1 & 45,0 & 0,047 & - \\
\hline
\end{tabular}

${ }^{1} \mathrm{dln}$ : degrés de liberté au numérateur.

${ }^{2} \mathrm{dld}$ : degrés de liberté au dénominateur (exemple pour la PAH).

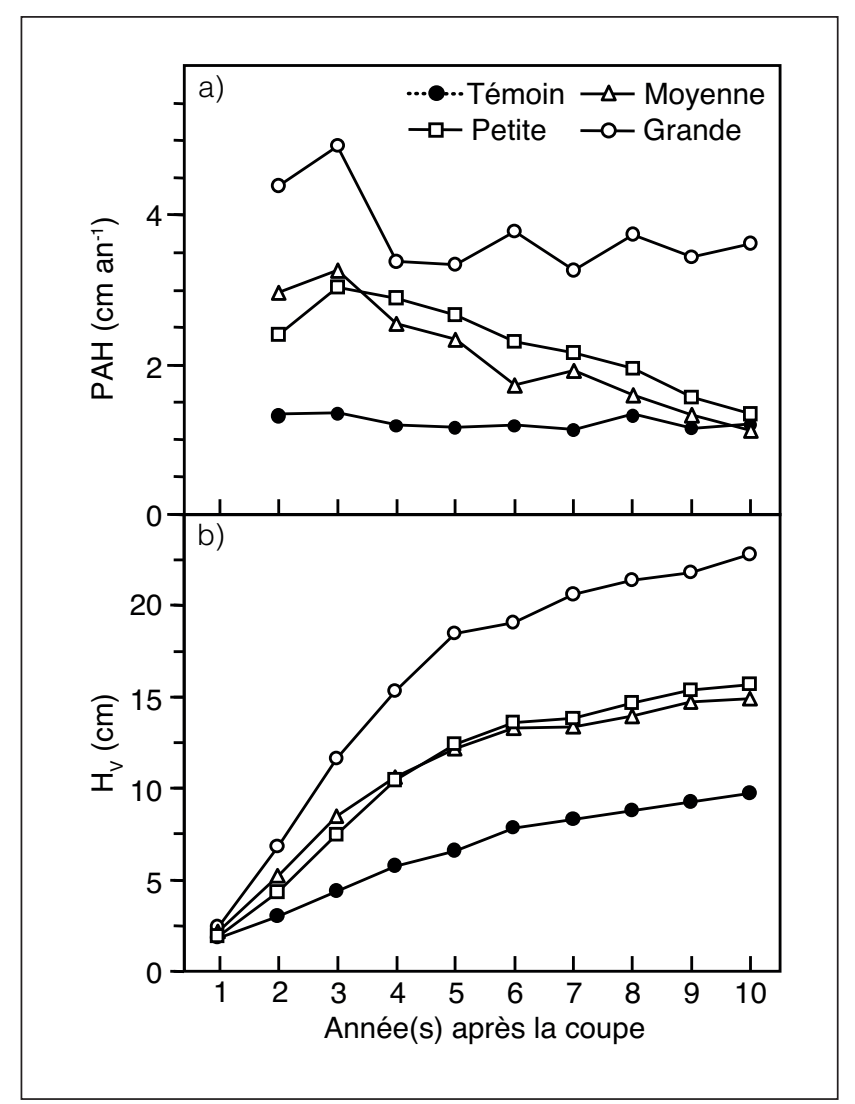

Fig. 5. Effets de la taille des trouées (définies au Tableau 1) et du temps sur a) la pousse annuelle en hauteur (PAH) et b) l'évolution de la hauteur $\left(\mathrm{H}_{v}\right)$ des semis d'épinette rouge. L'erreur-type maximale est de $\pm 0,2 \mathrm{~cm} \mathrm{an}^{-1}$ pour la $\mathrm{PAH}$ et de $\pm 2 \mathrm{~cm}$ pour la $\mathrm{H}_{\mathrm{v}}$.

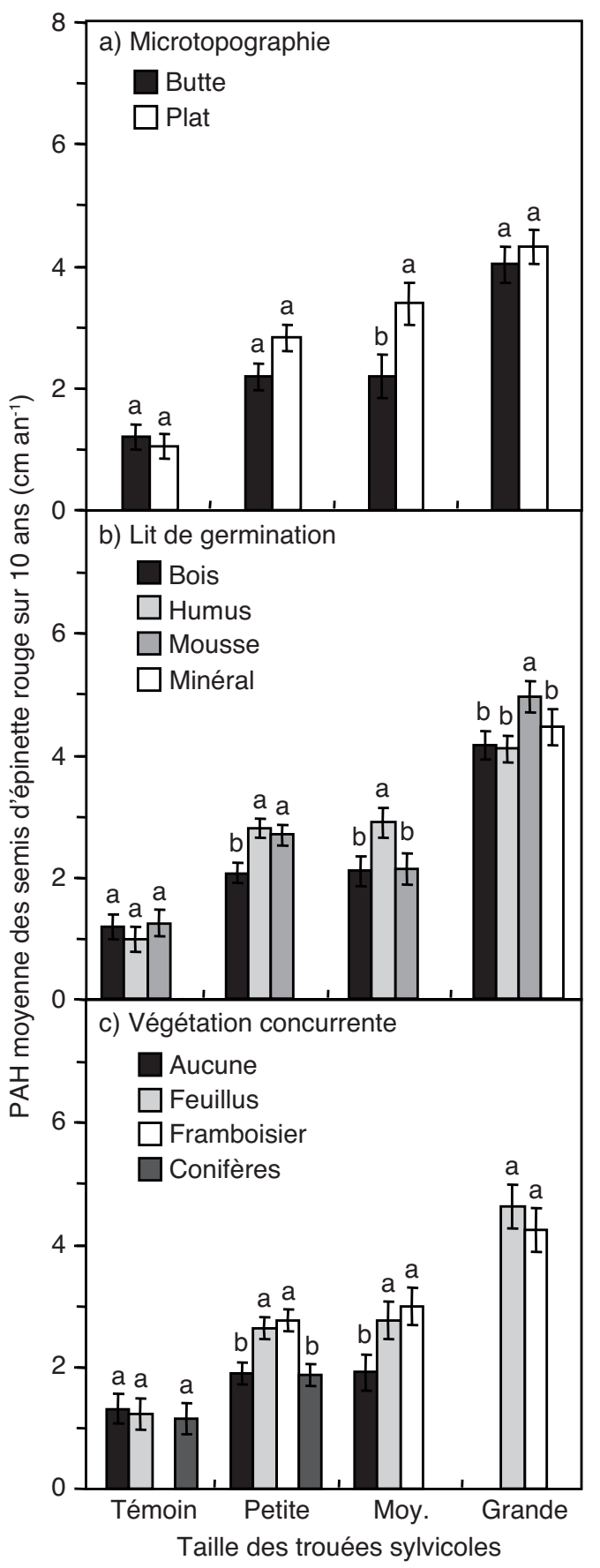

Fig. 6. Effets a) de la microtopographie, b) du lit de germination et c) de la végétation concurrente dominante sur la pousse annuelle en hauteur (PAH) des semis d'épinette rouge, selon la taille des trouées (définies au Tableau 1). Pour une même taille de trouée, les colonnes de l'histogramme ayant la même lettre ont des valeurs qui ne sont pas statistiquement différentes. Les barres verticales montrent l'erreur-type. 
trouées ait donc été occupée par d’autres espèces concurrentes ou aucune végétation, les semis d'épinette ont presqu'exclusivement été accompagnés par du framboisier (Fig. 3c). Comme lépinette rouge tolère mal les conditions extrêmes (Dumais et Prévost 2007), il se peut que le couvert de framboisier ait tempéré le microclimat dans l'entourage des semis (Man et Greenway 2011, Hyppönen et al. 2013).

Établissement (année 10), croissance et facteurs explicatifs Après la germination, les semis doivent survivre et se développer (phase détablissement), sans quoi leur avenir est incertain. Or, les conditions favorables à la germination et à la survie initiale (Pauley 1993) ne le sont pas toujours pour ce qui est de létablissement et de la croissance à moyen et long termes (Takahashi et al. 2000, Burgess et Wetzel 2002, Prévost et Dumais 2003). Dans cette étude, une densité initiale relativement élevée et une survie parmi les plus élevées ont permis aux petites trouées $<100 \mathrm{~m}^{2}$ d’offrir le meilleur établissement de semis, malgré une croissance modérée. À l'inverse, les conditions extrêmes dans le témoin et les grandes trouées de $700 \mathrm{~m}^{2}$ ont réduit la survie et par le fait même l'établissement dans ces niches de régénération hautement différentes. Les variables de croissance $\left(\right.$ Diam $_{c}, \mathrm{NBV}, \mathrm{PAH}$ et $\mathrm{H}_{\mathrm{v}}$ ) ont toutefois été supérieures dans les grandes trouées, là où la lumière était la plus disponible, confirmant notre troisième hypothèse et réitérant que l'épinette rouge a besoin, après sêtre établie, d'une hausse de luminosité pour optimiser sa croissance (Seymour 1995, Dumais et Prévost 2008, 2014, Greenwood et al. 2008).

Lanalyse des variables du milieu a permis d'identifier les conditions influençant le plus la survie et la croissance des semis dépinette rouge (hypothèse 2 ). Pour toutes les tailles de trouées, la survie a été meilleure sur les buttes (Fig. 4a). Ces dernières accumulent moins de litière et éloignent les semis de la végétation concurrente (voir Dumais et Prévost 2007). Par contre, dans les moyennes trouées de 100 à $300 \mathrm{~m}^{2}$, un meilleur accès à l'eau et aux éléments nutritifs combiné à des conditions acceptables de lumière et de température pourraient expliquer pourquoi les surfaces planes ont favorisé la croissance en hauteur (Fig. 6a).

Le lit de germination a aussi eu un impact sur létablissement. Dans les grandes trouées, exposant le plus la régénération au rayonnement solaire, la survie des semis a été nettement plus élevée sur le bois en décomposition que sur tout autre substrat (Fig. 4b). La grande rétention en eau de ce substrat peut expliquer la survie de lépinette malgré les conditions extrêmes d'évapotranspiration rencontrées dans les grandes trouées. Ce résultat vient renforcer l'hypothèse de Prévost et Charette (2015) stipulant que le manque de bois pourri soit en partie responsable de l'échec d'établissement des semis dépinette rouge dans cette niche de régénération. Toutefois, il faut mentionner que ce lit de germination bien pourvu en eau na pas permis une croissance en hauteur optimale dans la présente étude (Fig. 6b), possiblement en raison de la faible disponibilité des nutriments (Harmon et al. 1986).

En ce qui a trait à la végétation concurrente, nous avons étonnamment constaté que les feuillus et le framboisier, bien que ce dernier puisse aussi être néfaste aux jeunes conifères (Prévost et al. 2010, Prévost et Charette 2015), pouvaient avoir favorisé l'établissement de nouveaux semis dépinette rouge dans de petites ou moyennes trouées sylvicoles (Figs. 4c et $6 \mathrm{c}$ ). Tel que mentionné précédemment, l'effet d'abri que procure cette végétation, en limitant entre autres les variations du microclimat, pourrait expliquer ce résultat.

En accord avec notre dernière hypothèse, nos données montrent que le développement de nouveaux semis dépinette rouge est particulièrement lent. Dans les moyennes trouées de 100 à $300 \mathrm{~m}^{2}$, simulant le mieux la dynamique naturelle (Kneeshaw et Prévost 2007), la croissance en hauteur n'a été que de $2 \mathrm{~cm}$ an $^{-1}$. Malheureusement, à notre connaissance, aucune autre recherche n'a porté sur la croissance de semis naturels d'épinette rouge aussi jeunes, de sorte qu'il est difficile de comparer nos résultats.

\section{Synthèse, conclusions et implications pour la sylviculture}

La germination et létablissement de semis naturels d'épinette rouge dépendent du microenvironnement, lequel est influencé par la pratique sylvicole. Le degré d’ouverture du couvert forestier joue un rôle important à cet égard. Les petites trouées sylvicoles $<100 \mathrm{~m}^{2}$ ont été de loin plus favorables à la germination et à l'établissement que les trouées de 100-300 $\mathrm{m}^{2}$ ou $700 \mathrm{~m}^{2}$. Toutefois, il semble que ces plus grandes trouées soient éventuellement nécessaires à la croissance optimale des semis, militant en faveur d'un scénario de coupe progressive irrégulière (voir Raymond et al. 2009). En outre, la baisse inquiétante de survie sur 10 ans (Fig. 2b) lance un appel à la prudence lors d'inventaires de régénération précoces après coupe afin de ne pas surévaluer la densité potentielle de semis d'épinette rouge.

Létude confirme le rôle prépondérant du bois en décomposition et de la mousse pour la germination et l'établissement de l'espèce sous couvert. La présence de bois pourri s'est même avérée nécessaire à l'établissement dans les grandes trouées, alors que l'humus a été plus favorable dans les moyennes trouées. Dans les petites trouées, où les conditions semblaient plus homogènes, les germinants de même que les semis établis se sont retrouvés autant sur de la mousse que sur de l'humus ou du bois en décomposition.

En somme, pour maximiser la régénération naturelle de l'épinette rouge, les sylviculteurs devraient créer de petites trouées combinées à une rétention de bois mort à différents stades de décomposition, notamment si l'agrandissement des trouées est envisagé. Vu les faibles densités de semis que nous avons observées et leur croissance modeste, la plantation dans de petites ou moyennes trouées sylvicoles est une avenue à explorer afin d'accélérer le renouvellement de l'espèce, d'autant plus si la régénération préétablie est déficiente.

\section{Remerciements}

Nous tenons à remercier deux réviseurs anonymes pour leurs commentaires et suggestions qui ont permis de bonifier ce document. Le travail technique de Julie Forgues, Carlo Gros-Louis, Daniel Guimond, Serge Williams, Pascal Lainé, Étienne Du Berger et plusieurs étudiants a aussi été grandement apprécié. Enfin, nous sommes reconnaissants envers Josianne DeBlois pour ses conseils lors des analyses statistiques. Cette recherche a été financée dans le cadre des projets 142332016 et 142332111 de la Direction de la recherche forestière (Ministère des Forêts, de la Faune et des Parcs du Québec). 


\section{Références}

Archambault, L., J. Bégin, C. Delisle et M. Fortin. 2003. Dynamique forestière après coupe partielle dans la Forêt expérimentale du Lac Édouard, Parc de la Mauricie, Québec. Forest. Chron. 79: 672-684.

Burgess, D. et S. Wetzel. 2002. Recruitment and early growth of eastern white pine (Pinus strobus) regeneration after partial cutting and site preparation. Forestry 75: 419-423.

Cavallin, N. et L. Vasseur. 2008. Potential for red spruce (Picea rubens Sarg.) establishment from natural seed dispersal in old fields adjacent to forest stands. Plant Ecol. 199: 33-41.

Dibble, A.C., J.C. Brissette et M.L. Hunter Jr. 1999. Putting community data to work: Some understory plants indicate red spruce regeneration habitat. For. Ecol. Manage. 114: 275-291.

Dumais, D. et M. Prévost. 2007. Management for red spruce conservation in Québec: The importance of some physiological and ecological characteristics - A review. Forest. Chron. 83: 378-392.

Dumais, D. et M. Prévost. 2008. Ecophysiology and growth of advance red spruce and balsam fir regeneration after partial cutting in yellow birch-conifer stands. Tree Physiol. 28: 1221-1229.

Dumais, D. et M. Prévost. 2013. Lépinette rouge (Picea rubens Sarg.). Dans Le guide sylvicole du Québec (tome 1) : Les fondements biologiques de la sylviculture. Ouvrage collectif sous la supervision de B. Boulet et M. Huot, Min. Res. Nat. Québec. Les Publications du Québec, p. 128-131.

Dumais, D. et M. Prévost. 2014. Physiology and growth of advance Picea rubens and Abies balsamea regeneration following different canopy openings. Tree Physiol. 34: 194-204.

Dwyer, L.M. et G. Merriam. 1981. Influence of topographic heterogeneity on deciduous litter decomposition. Oikos 37: 228-237.

Farrar, J.L. 1996. Les arbres du Canada. Les Éditions Fides, 502 p.

Frank, R.M. et B.M. Blum. 1978. The selection system of silviculture in spruce-fir stands - Procedures, early results, and comparisons with unmanaged stands. For. Serv. Res. Pap. NE-425, USDA For. Serv., Northeast. For. Exp. Stat., Broomall, PA, 16 p.

Fraver, S., R.G. Wagner et M. Day. 2002. Dynamics of coarse woody debris following gap harvesting in the Acadian forest of central Maine, U.S.A. Can. J. For. Res. 32: 2094-2105.

Greenwood, M.S., C.L. O'Brien, J.D. Schatz, C.A. Diggins, M.E. Day, G.L. Jacobson, A.S. White et R.G. Wagner. 2008. Is early life cycle success a determinant of the abundance of red spruce and balsam fir? Can. J. For. Res. 38: 2295-2305.

Grondin, P. et A. Cimon. 2003. Les enjeux de biodiversité relatifs à la composition forestière. Min. Res. Nat. Faune Parcs Québec, Sainte-Foy, QC, 200 p.

Harmon, M.E., J.F. Franklin, F.J. Swanson, P. Sollins, S.V. Gregory, J.D. Lattin, N.H. Anderson, S.P. Cline, N.G. Aumen, J.R. Sedell, G.W. Lienkaemper, K. Cromack et K.W. Cummins. 1986. Ecology of coarse woody debris in temperate ecosystems. Adv. Ecol. Res. 15: $133-302$.

Hyppönen, M., V. Hallikainen, J. Niemelä et P. Rautio. 2013. The contradictory role of understory vegetation on the success of Scots pine regeneration. Silva Fenn. 47 article id 903.19 p.

Kenefic, L.S., M. Bataineh, J.S. Wilson, J.C. Brissette et R.D. Nyland. 2014. Silvicultural rehabilitation of cutover mixedwood stands. J. For. 112: 261-271.

Klein, R.M., T.D. Perkins, J. Tricou, A. Oates et K. Cutler. 1991. Factors affecting red spruce regeneration in declining areas of Camels Hump Mountain, Vermont. Am. J. Bot. 78: 1191-1198.

Kneeshaw D.D. et M. Prévost. 2007. Natural canopy gap disturbances and their role in maintaining mixed-species forests of central Quebec, Canada. Can. J. For. Res. 37: 1534-1544.

Man, R. et K.J. Greenway. 2011. Effects of artificial shade on early performance of white spruce seedlings planted on clearcuts. New For. 41: 221-233.

Morgenstern, E.K. et J.L. Farrar. 1964. Introgressive hybridization in red spruce and black spruce. For. Tech. Bull. 4, University of Toronto, Faculty of forestry, Toronto, ON, $47 \mathrm{p}$.
MDDELCC (Ministère du développement durable, de l'environnement et de la lutte contre les changements climatiques). 2015. Normales climatiques du Québec 1981-2010. Station Honfleur (www.mddelcc.gouv.qc.ca/climat/normales).

Nyland, R.D. 2002. Silviculture: Concept and Applications, Deuxième édition. McGraw-Hill, New York, 682 p.

Örlander, G., P. Gemmel et J. Hunt. 1990. Site preparation: A Swedish overview. For. Can. and British Columbia Min. For. FRDA Rep. 105, $61 \mathrm{p}$.

Pauley, E.F. 1993. The influence of episodic reproduction by red spruce on long-term forest dynamics in the Cranberry Wilderness, West Virginia. Ph.D. Dissertation, Univ. of Tennessee, Knoxville, $167 \mathrm{p}$.

Perkins, T.D. 1991. Gap expansion, seedling regeneration, and microclimate in a disturbed montane red spruce - balsam fir forest in northern Vermont. Ph.D. Diss., Univ. of Vermont, Burlington, VT. Place, I.C.M. 1955. The influence of seed-bed conditions on the regeneration of spruce and balsam fir. Bull. 117, Can. Dept. North. Aff. Nat. Res., For. Br., For. Res. Div., 87 p.

Prévost, M. et L. Charette. 2015. Selection cutting in a yellow birch-conifer stand in Quebec, Canada: Comparing the single and two hybrid methods using different sizes of canopy opening. For. Ecol. Manage. 357: 195-205.

Prévost, M. et D. Dumais. 2003. Croissance et statut nutritif de marcottes, de semis naturels et de plants dépinette noire à la suite du scarifiage : résultats de 10 ans. Can. J. For. Res. 33: 2097-2107.

Prévost, M. et D. Pothier. 2003. Partial cuts in a trembling aspen conifer stand:

Effects on microenvironmental conditions and regeneration dynamics. Can. J. For. Res. 33: 1-15.

Prévost, M., P. Raymond et J.-M. Lussier. 2010. Regeneration dynamics after patch cutting and scarification in yellow birch - conifer stands. Can. J. For. Res. 40: 357-369.

Raymond, P., S. Bédard, V. Roy, C. Larouche et S. Tremblay. 2009. The irregular shelterwood system: Review, classification, and potential application to forests affected by partial disturbances. J. For. 107: 405-413.

Robert, E., S. Brais, B.D. Harvey et D. Greene. 2012. Seedling establishment and survival on decaying logs in boreal mixedwood stands following a mast year. Can. J. For. Res. 42: 1446-1455.

Ruel, J.-C., D. Lousteau et M. Pineau. 1988. Relations entre la microtopographie, les caractéristiques de la couverture morte et la répartition des essences dans une érablière à Bouleau jaune. Can. J. For. Res. 18: 1196-1202.

Seymour, R.S. 1995. The Northeastern region. Dans J.W. Barrett (éditeur). Regional silviculture of the United States, $3^{\text {ème }}$ édition. Wiley and Sons, New York, p. 31-79.

Smallidge, P.J. et D.J. Leopold. 1994. Forest community composition and juvenile red spruce (Picea rubens) age-structure and growth patterns in an Adirondack watershed. Bull. Torrey Bot Club 121: 345-356.

Takahashi M., Y. Sakai, R. Ootomo et M. Shiozaki. 2000. Establishment of tree seedlings and water-soluble nutrients in coarse woody debris in an old-growth Picea abies forest in Hokkaido, northern Japan. Can. J. For. Res. 30: 1148-1155.

Weaver, J.K., L.S. Kenefic, R.S. Seymour et J.C. Brissette. 2009. Decaying wood and tree regeneration in the Acadian Forest of Maine, USA. For Ecol. Manage. 257: 1623-1628.

Westveld, M. 1953. Ecology and silviculture of the spruce-fir forests of eastern North America. J. For. 51: 422-430.

White, P.S. et C.V. Cogbill. 1992. Spruce-fir forests of eastern North America. Dans C. Eagar et M.B. Adams (éditeurs). Ecology and decline of red spruce in the eastern United States, p. 3-39. Ecological Studies 96, Springer-Verlag, New York. 